\title{
Analysis of clinical outcome and postoperative organ function effects in a propensity-matched comparison between conventional and minimally invasive mitral valve surgery
}

\author{
Sophie Missault ${ }^{1}$, Jerome Van Causenbroeck ${ }^{1}$, Korneel Vandewiele ${ }^{1}$, Jens Czapla ${ }^{1}$, Tine \\ Philipsen $^{1}$, Katrien François ${ }^{2}$, and Thierry Bove ${ }^{3}$ \\ ${ }^{1}$ University Hospital Ghent \\ ${ }^{2}$ University of Ghent \\ ${ }^{3}$ University Hopsital of Ghent
}

July 24, 2020

\begin{abstract}
Background: Minimally invasive mitral valve(MV) surgery(MIVT) is increasingly performed with excellent clinical outcome, despite longer procedural times. This study analyzes clinical outcome and secondary organ function effects in a propensity-matched comparison with conventional MV surgery. Methods and Results: Out of 439 patients undergoing MV surgery from January 2005 to May 2017, 345 patients were included after propensity-matching: 95 sternotomy patients and 250 MIVT patients. Endpoints focused on survival, quality of MV repair and organ function effects through analysis of biomarkers and functional parameters. Despite longer cardiopulmonary bypass(sternotomy: 96.0(IQR34)min - MIVT:134.0(IQR42)min, p<0.001) and cardioplegic arrest times(sternotomy: 61.0(IQR26)min - MIVT:87.0(IQR34)min, p<0.001), no differences in survival nor complication rate were found. Effect on renal function(creatinine, $\mathrm{p}=0.751$ - ureum, $\mathrm{p}=0.538$ - glomerular filtration, $\mathrm{p}=0.848$ ), myocardial damage by troponine I level (sternotomy: $1.8 \pm 3.9 \mathrm{ng} / \mathrm{ml}-\mathrm{MIVT}: 1.2 \pm 1.3 \mathrm{ng} / \mathrm{ml}, \mathrm{p}=0.438$ ) and ventilatory support $>24$ hours(sternotomy:5.5\% - MIVT:9.5\%, p=0.240) were comparable. Systemic inflammatory reaction by postoperative CRP count was markedly lower for $\operatorname{MIVT}(\mathrm{p}<0.001)$. Increased rhadomyolysis was found after MIVT surgery, based on significant elevation of creatinine-kinase levels(sternotomy: 431 $\pm 237 \mathrm{U} / \mathrm{L}-\mathrm{MIVT}$ : 701 $\pm 595 \mathrm{U} / \mathrm{L}, \mathrm{p}<0.001)$. Conclusion: Despite an inherent learning curve, minimally invasive MV surgery guarantees a clinical outcome and MV repair quality, at least non-inferior to those of MV surgery via sternotomy. Notwithstanding longer cardiopulmonary bypass and cardiac arrest times, the impact on secondary organ function is negligible, excepted for a lower systemic inflammatory response. The postoperative increase of CK-enzymes suggestive for enhanced rhabdomyolysis needs to be accounted when procedural times tend to exceed the critical time threshold for severe limb ischemia.
\end{abstract}

\section{Analysis of clinical outcome and postoperative organ function effects in a propensity-matched comparison between conventional and minimally invasive mitral valve surgery}

Sophie Missault MD. ${ }^{1^{*}}$, Jérôme Van Causenbroeck MD..$^{*}$, Korneel Vandewiele MSc. ${ }^{2}$, Jens Czapla MD. ${ }^{1}$, Tine Philipsen MD. ${ }^{1}$, Katrien François MD.PhD. ${ }^{1}$, Thierry Bové MD.PhD. ${ }^{1}$

${ }^{1}$ Department of Cardiac Surgery ${ }^{1}$ and Perfusion ${ }^{2}$

University Hospital of Ghent, Ghent, Belgium

*Both authors contributed equally in the submission of this manuscript

Corresponding author: 
Thierry Bové MD. PhD.

Department of Cardiac Surgery

University Hospital Gent

Corneel Heymanslaan 10, 9000 Gent, Belgium

Phone: +32-93323925

E-mail: thierry.bove@ugent.be

Funding: None

Short running title: Port-access Mitral Valve Surgery

Word count:

Abstract: 250 - Manuscript: 4495

Abstract

Background:

Minimally invasive mitral valve(MV) surgery(MIVT) is increasingly performed with excellent clinical outcome, despite longer procedural times. This study analyzes clinical outcome and effect on secondary organ functions in a propensity-matched comparison with conventional MV surgery.

Methods and Results:

Out of 439 patients undergoing MV surgery from January 2005 to May 2017, 345 patients were included after propensity-matching: 95 sternotomy patients and 250 MIVT patients. Endpoints focused on survival, quality of MV repair and organ function effects through analysis of biomarkers and functional parameters.

Regardless of longer cardiopulmonary bypass(sternotomy: 96.0(IQR34)min - MIVT:134.0(IQR42)min, $\mathrm{p}<0.001$ ) and cardioplegic arrest times(sternotomy: 61.0(IQR26)min - MIVT:87.0(IQR34)min, $\mathrm{p}<0.001$ ), no differences in survival nor complication rate were found. Effect on renal function(creatinine, $\mathrm{p}=0.751$ - ureum, $\mathrm{p}=0.538$ - glomerular filtration, $\mathrm{p}=0.848$ ), myocardial damage by troponine I level (sternotomy: $1.8 \pm 3.9 \mathrm{ng} / \mathrm{ml}$ - MIVT:1.2 $\pm 1.3 \mathrm{ng} / \mathrm{ml}, \mathrm{p}=0.438$ ) and prolonged ventilatory support $>24$ hours(sternotomy:5.5\% MIVT:9.5\%, $\mathrm{p}=0.240$ ) were comparable. Systemic inflammatory reaction by postoperative CRP count was markedly lower for MIVT $(\mathrm{p}<0.001)$. Increased rhadomyolysis was found after MIVT surgery, based on significant elevation of creatinine-kinase levels(sternotomy: 431 $\pm 237 \mathrm{U} / \mathrm{L}-\mathrm{MIVT}$ : $701 \pm 595 \mathrm{U} / \mathrm{L}, \mathrm{p}<0.001$ ).

\section{Conclusion:}

Despite an inherent learning curve, minimally invasive MV surgery guarantees a clinical outcome and MV repair quality, at least non-inferior to those of MV surgery via sternotomy. Notwithstanding longer cardiopulmonary bypass and cardiac arrest times, the impact on secondary organ function is negligible, excepted for a lower systemic inflammatory response. The postoperative increase of CK-enzymes suggestive for enhanced rhabdomyolysis needs to be accounted when procedural times tend to exceed the critical time threshold for severe limb ischemia.

\section{Introduction}

The concept of a minimally invasive approach for mitral valve(MV) surgery has been initiated in the nineties by Cosgrove et al., and was primarily based on attempts to reduce the surgical invasiveness by limiting the incision length and avoiding a full sternotomy ${ }^{1,2}$. Encouraged by advances in thoracic and abdominal surgery, an endoscopic approach with thoracoscopic visualization and use of peripheral cardiopulmonary bypass, aided to minimize the surgical trauma ${ }^{3}$. Meanwhile, this 'port-access' surgery has become the preferred technique for mitral and/or tricuspid valve surgery in many centers. Several studies pointed repetitively to the obvious benefits as an improved cosmetic result, a shorter hospital stay, quicker socio-economic reintegration 
and decreased need for blood products, whilst the main surgical end-points in terms of quality of mitral repair and morbidity as mortality were commonly maintained once the inevitable learning curve has been surpassed $^{4-11}$. Hence, even in experienced hands, port-access MV surgery is associated with longer duration of cardiopulmonary bypass and aortic cross-clamp time, known of having an adverse effect on postoperative morbidity and mortality due to secondary organ dysfunction ${ }^{5,6,8}$.

The purpose of this study is to compare the clinical outcome of MV surgery performed through port-access and conventional sternotomy in a propensity-matched cohort, with additional focus on secondary organ function by analysis of organ-specific biomarkers and/or other functional outcome parameters.

\section{Material and methods}

This study was approved by the ethical committee of the University Hospital of Ghent, waiving patient's informed consent by the retrospective study design and the use of anonymized data (reference B67201732290/91).

\section{Surgical technique of minimally invasive videoscopic MV surgery (MIVT)}

The MIVT program at our institution started in 2008, and has been applied to all patients presenting for elective MV surgery since then. Patients were omitted during the early phase of the program in case of previous cardiac surgery, extensive annular calcifications and precarious vascular access. Preoperative screening for MIVT systematically included angiographic imaging of the complete aorta to femoral vessels, to rule out severe atherosclerotic disease. Patients were routinely intubated with a double-lumen endotracheal tube to allow single lung ventilation. Surgical access was obtained through a right anterior thoracotomy at the $4^{\text {th }}$ intercostal space, via a 5-6 cm skin incision. Cardiopulmonary bypass was installed through cannulation of the femoral vein and artery via Seldinger technique, and correct positioning of the cannulas under guidance of transesophageal echocardiography. Cardioplegic arrest was achieved by antegrade administration of cold crystalloid cardioplegia in the aortic root after aortic clamping with Chitwood clamp or endoaortic balloon occlusion (IntraClude, Edwards Lifesc., Irvine, CA, USA). MV repair was done according to classical techniques.

MV surgery through sternotomy was used as standard approach before the end of 2008, and was still used later on for patients requiring concomitant procedures like aortic valve surgery or coronary artery bypass grafting, patients with extensive mitral annular calcifications, or those presenting with fulminant lung edema in need for preoperative respiratory support, prohibiting single lung ventilation.

\section{Patient data}

From January 2005 to May 2017, 439 patients underwent MV surgery at our department. A MIVT procedure was done in 250 patients between July 2008 and May 2017, and 189 patients were operated through conventional sternotomy between January 2005 and December 2014. Pre-, intra- and postoperative data were retrospectively retrieved from the patient's medical records. Organ-specific biomarkers were obtained preoperatively and per 24 hour on ICU concerning assessment of liver, kidney and myocardial damage, while lung and brain function were based on clinical parameters such as ventilation time and stroke incidence respectively. Evaluation of systemic inflammatory response was based on CRP values, obtained preoperatively and at the first and second day after surgery. Use of blood products, postoperative blood loss and hematological assessment were additionally included.

The clinical follow-up ended in November 2017, considering the endpoints survival, reoperation free-survival and late MV function.

\section{Statistical Analysis}

Statistical analysis was performed using SPSS Statistics 25.0 (IBM SPSS Statistics for Windows, Version 25.0, Armonk, NY).

Since both surgical procedures were performed consecutively over time yielding only a limited time overlap during the learning phase of MIVT, this study is inherently biased. Therefore, a propensity-score matched 
analysis with a tolerance of 0.05 and without replacement was executed for the patient characteristics having a baseline significant difference. Adjustment was performed for differences in preoperative NYHA class and previous cardiac surgery to match both study groups.

Data distribution is verified for normality by the Shapiro-Wilk test. Categorical variables are expressed by number and percentage. Continuous variables are noted as mean and standard deviation or as median and interquartile range, depending on the distribution normality of the data. Categorical variables were compared between groups by Chi-square or Fisher's exact test. Comparison of continuous data was done by the unpaired t-test or Mann-Whitney U-test.

To examine significant between-group differences in biomarker evolution over time points, an repeated ANOVA-analysis was used. Significance was based on Wilk's-Lambda or Greenfeld-Houser testing depending on Mauchly's sphericity preservation of the data. Survival analysis was performed by the Kaplan-Meier estimation method with log-rank testing of the difference between both procedures. All tests were performed 2 -tailed and considered as statistically significant for a p-value $<0.05$.

Results

\section{Patient demographics}

In the entire cohort of 439 patients, the sternotomy group $(n=189)$ showed a higher NYHA class $(\mathrm{p}<0.001)$ and underwent more frequently prior cardiac surgery (sternotomy: $26.5 \%$ vs. MIVT: $4.8 \%, \mathrm{p}<0.001$ ). After propensity-score matching for these variables, 345 patients were retained for analysis, respectively 95 in the sternotomy group and 250 patients in the MIVT group, showing comparable demographics(Table 1).

\section{Operative characteristics}

The operative data are shown in table 2. Regarding the etiology of MV disease, isolated annular dilation was noticed more frequently in the MIVT group $(28.5 \%$ versus $8.7 \%, \mathrm{p}<0.001)$, in contrast to the incidence of degenerative MV disease slightly in favor of the sternotomy group (sternotomy: $72.8 \%$ versus MIVT: $59.4 \%$, $\mathrm{p}=0.054$ ) (figure 1). Accordingly, the number of mitral valve replacements was higher in the sternotomy group, while isolated annuloplasty was performed more frequently in the MIVT group. No significant differences were found regarding the use of neochordae and the performance of concomitant AF ablation or tricuspid surgery. Duration of cardiopulmonary bypass and aortic cross-clamp time were significantly longer in the MIVT group.

\section{Postoperative morbidity and mortality}

There was no difference in major complications rate in terms of stroke rate, pulmonary complications, and acute renal failure and/or need for dialysis. New-onset atrial fibrillation occurred in $4.1 \%$ of MIVT patients versus $7.8 \%$ of the sternotomy patients $(\mathrm{p}=0.259)$, after exclusion of the confounding effect of concomitant ablation therapy. A significantly shorter hospital stay was noticed in the MIVT group (median: 13(IQR 4) d vs. $14(\mathrm{IQR} 4) \mathrm{d}, \mathrm{p}=0.016$ ), while ICU stay was comparable for both groups (sternotomy: 1.4(IQR 1.8) d vs. MIVT: $1.3($ IQR1.7) d, $\mathrm{p}=0.366)$.

Seven MIVT patients needed intra-operative conversion to median sternotomy for respectively traumatic laceration of the left atrial appendage by the Chitwood $\operatorname{clamp}(n=2)$, retrograde aortic dissection $(n=1)$, bleeding of the right ventricle during pacemaker wire insertion(n=1), circumflex artery occlusion( $\mathrm{n}=1)$, and final need for mitral valve replacement after prolonged attempts of MV repair(n=2).

In-hospital mortality was similar for both groups: MIVT: $\mathrm{n}=5(2.0 \%)$ patients versus sternotomy: $\mathrm{n}=1(1.1 \%)(\mathrm{p}=1.000)$.

\section{Late outcomes}

Clinical follow-up was complete for 302(87.5\%) patients of the whole cohort (sternotomy: $75.8 \%$ versus MIVT: $92.0 \%, \mathrm{p}<0.001)$. Median follow-up duration was 3.1(IQR 4.2)y, and significantly longer in the sternotomy 
group (sternotomy: 6.5(IQR 6)y versus MIVT: 2.5(IQR 4.3)y, $\mathrm{p}<0.001)$. The cumulative patient follow-up time was 962 patient/years.

Kaplan-Meier analysis showed no significant survival difference between the groups $(\mathrm{p}=0.472)$ (Figure 2). At last follow-up, sternotomy patients showed a higher NYHA class $(\mathrm{p}=0.015)$ and appeared to develop more frequently atrial fibrillation (Sternotomy: $3.8 \%$ vs. MIVT: $15.7 \%, \mathrm{p}=0.002$ ).

Echocardiographic assessment of MV function at last follow-up showed a similar quality of MV repair for the 2 groups, in terms of freedom from regurgitation(figure 3) as well as transmitral gradient (mean gradient: sternotomy 4.0(2.0) $\mathrm{mmHg}$ versus MIVT 4.0 (2.0) $\mathrm{mmHg}, \mathrm{p}=0.810)$; max gradient: sternotomy 10.0(6.8) $\mathrm{mmHg}$ versus MIVT 10.0(5.4) $\mathrm{mmHg}, \mathrm{p}=0.957)$.

Late reoperation for recurrent MV dysfunction was necessary in 8(8.6\%) sternotomy patients vs. 11(4.4\%) MIVT patients $(\mathrm{p}=0.130)$, revealing a comparable freedom from MV-related reoperation of $90.8 \pm 3.6 \%$ and $90.8 \pm 3.6 \%$ in the sternotomy group versus $93.9 \pm 2.0 \%$ and $90.7 \pm 3.0 \%$ in the MIVT group at respectively 3 and 5 years $($ logrank $\mathrm{p}=0.529)$ (Figure 4$)$.

Organ-specific biomarker analysis (Table 4)

Analysis of liver function biomarkers showed an AST count increase in both groups, but significantly higher in MIVT, together with raised ALT counts. In contrast, LDH levels increased significantly more in the sternotomy group compared to MIVT group.

Renal function biomarkers increased similarly in both groups as shown in Figure 5, corresponding with a decrease of GRF, but without reaching a statistically significant difference.

Myocardial damage assessed by troponin I and creatine kinase(CK) and creatine kinase MB(CK-MB) counts revealed no difference of postoperative troponin levels between MIVT and sternotomy. However, CK counts increased significantly more in the MIVT group than in the sternotomy group (MIVT: 89.74 to 933.02 U/1 vs. sternotomy: 73.92 to $89.74 \mathrm{U} / \mathrm{l} ; \mathrm{p}=0.002$ ). In contrast, CK-MB levels increased postoperatively in both groups, albeit based on samplings of only 44 patients.

Non-specific systemic inflammation was considered by leukocyte counts and C-reactive protein(CRP) levels. Leukocyte counts increased significantly more in MIVT compared to sternotomy. However, CRP counts raised significantly more from the first to second postoperative day in the sternotomy compared to the MIVT group $(\mathrm{p}<0.001)$ (Figure 6). Other hematologic biomarkers are listed in Table 4.

As red blood cell derivatives are confounded by intra- and postoperative transfusion, only the latter parameters are analyzed. Mediastinal drainage loss during the first 12 and 48 hours was higher in the MIVT group. No significant difference was found in the number of blood transfusions nor total blood transfusion volume.

The impact of the surgical approach on lung function was represented by the length of mechanical ventilation. No difference between groups was found concerning mechanical ventilation longer than 24 hours.

Discussion

Port-access MV surgery is nowadays a valid approach to treat all kinds of MV disease according to contemporary techniques, resulting in a clinical outcome equal to the conventional sternotomy approach. This technique has been implemented in our department since more than 10 years now for the surgical management of most MV pathologies, with or without the need for additional treatment of associated lesions as atrial fibrillation and tricuspid valve dysfunction. Even after including the obligatory learning phase, the clinical outcome as well as the quality of MV procedure itself appeared to be non-inferior to that of the standard MV surgery. Hence, the effect of the learning period during the adoption of a new approach is not negligible. In a large volume center, Holzhey et al. demonstrated that approximately 75 to 125 operations are required to achieve optimal results with MIVT, characterized by a net decrease of bleeding complications, reoperation for early valve failure and a nearly obsolete conversion rate ${ }^{12}$. 
Moreover, some complications are typically related to the surgical approach. The risk of stroke is often estimated to be increased due to the retrograde systemic perfusion and the potential risk for aortic dissection, inadequate aortic de-airing and the deficient tactile manipulation of the ascending aorta, regardless of the mode of aortic clamping ${ }^{13-14}$. The incidence of major neurological injury in our study was similar to others, revealing only one case of retrograde aortic dissection. Here, we underscore the importance of examining the vascular access - specifically the arterial side - rigorously to limit such devastating complication as much as possible.

Considering the lower traumatic impact of a mini-thoracotomy on the thorax compared to a sternotomy, one would expect a favorable effect on respiratory dynamics and so, shorter ventilation time. In this propensitymatched comparison, the ventilation times were identical as well as the ICU stay duration. In an equivalent study design comparing 350 port-access patients and 365 sternotomy patients, Suri et al. found a slight decrease of ventilatory support duration despite of significantly longer procedural times ${ }^{15}$. However, it is commonly known that the criteria for extubation may vary in-between centers, and that even in one single center, the decision for extubation may vary among ICU physicians, advancing thereby the subjective aspect of this parameter.

A recurrent finding of many studies on this topic is the notification of longer aortic cross-clamp and cardiopulmonary bypass times ${ }^{5-11}$. The adverse relationship between these factors and secondary organ dysfunction is well-known in the field of cardiac surgery ${ }^{16-17}$. Through analysis of specific organ function biomarkers, routinely sampled at the postoperative stage, we found no significantly different effect between both approaches. Biomarkers of myocardial and liver origin increased similarly in both groups, but one has to consider that, through lack of specificity, it is difficult to know what the real impact of each procedure on respectively myocardial and liver function is. Interestingly, the creatinine-kinase level was significantly increased after port-access surgery. Bearing in mind that this count also represents global muscle damage, one can relate this to a condition of relative limb ischaemia initiated by the cannulation of the femoral artery and vein. As the duration of cardiopulmonary bypass is rarely exceeding the critical duration threshold for irreversible ischemia and rhabdomyolysis, it generally remains clinically insidious. However, this issue needs to be accounted for optimization of the peripheral cannulation strategy, by using specifically designed arterial cannulas allowing distal leg perfusion or by avoiding combined ipsilateral femoral artery and vein cannulation.

Kidney biomarkers are both specific and sensitive to renal function. Our study demonstrated a rise of creatinine and ureum level, together with a decrease of glomerular filtration, during the early postoperative period, but independent of the used surgical approach. Nevertheless, the incidence of effective kidney failure, requiring renal replacement therapy, was low. Data on this organ-specific outcome are controversial, some showing a clear advantage for the minimally invasive technique, while others were not able to found any effect $^{15,18}$. One part of this confounding is related to the use of different study designs including selection bias and other statistical methods to analyze renal outcome.

Regarding the systemic inflammatory reaction, CRP counts remained largely inferior during the first 48 hours after port-access MV surgery than after conventional sternotomy. This suggests that the MIVT technique is associated with a significant trauma reduction to the body, with less activation of inflammatory mediators. Watt et al. reported similar results in a systematic review on CRP post-surgery, and identified that CRP counts were higher after bigger, more invasive surgical procedures like thoracic surgery compared to smaller surgeries such as cholecystectomy ${ }^{19}$. The results of Paparella et al. confirmed that CRP and interleukine-6 counts were lower after MIVT compared to sternotomy MV surgery ${ }^{20}$.

The lack of detrimental effect of longer duration of cardiopulmonary bypass and cardiac arrest on secondary organ function, as observed after port-access MV surgery, points to eventual intra-operative measures, directly related to the management of cardiopulmonary bypass. The conduct of cardiopulmonary bypass during MIVT surgery is commonly facing flow restrictions, due to the use of smaller caliber cannulas via peripheral vascular access, which on top of it, need to be properly positioned, depending on adequate guidance via intra-operative transesophageal echocardiography. Using kidney function as most robust organ function endpoint, our group recently demonstrated that an equivalent organ-specific as well as clinical outcome 
between MIVT and conventional MV surgery is achievable through targeting an minimal oxygen delivery during the duration of cardiopulmonary bypass. However, in order to cope with the frequent inferences of intra-operative flow restrictions over a longer procedural time during MIVT, other means need to be applied as the tolerance of lower body temperature, the pursuit of a higher intra-operative hematocrit level and the use of blood preserving measures ${ }^{21}$. Moreover, operative times may probably be less decisive for patient outcomes, as long as they are not excessively long.

\section{Limitations}

The limitations of this study are linked to its retrospective design, yielding missing data and incomplete follow-up. Regarding the validity of biomarkers to represent organ function, the low specificity of some biomarkers might impede clear interpretation of some data, as for example, liver enzymes AST, ALT and $\mathrm{LDH}$ are known to have other tissue sources. Therefore, this study would have been favored by a prospective design, incorporating the follow-up of organ-specific markers.

Since MIVT was used consecutively for MV surgery since 2009, with a short time overlap to cover the learning phase, there is a historical difference between the study groups. This might include some selection bias, where patients with more complex MV pathology were preferably operated through conventional approach during the transit period. As we found it mandatory to include the learning period, propensity-matching was performed for patient-related conditions but not for MV disease. However, we feel confident that even in a fully 1:1-matched cohort, the clinical outcome and organ function results would be comparable, demonstrating that the port-access approach allows to achieve a qualitatively identical outcome despite longer procedural times.

\section{Conclusion}

Minimally invasive port-access surgery is an equivalent alternative to the conventional median sternotomy approach for the treatment of all-kind of MV diseases, but offers an improved cosmetic result and faster postoperative recovery as most important advantage. Despite an inherent learning curve, this technique provides to achieve the same results in terms of valve repair quality and clinical outcome. Notwithstanding the commonly longer cardiopulmonary bypass and cardiac arrest times, the impact on secondary organ function is comparable to conventional median sternotomy, excepted for the lower systemic inflammatory response by CRP counts. Hence, the postoperative increase of CK-enzymes suggestive for enhanced rhabdomyolysis needs to be accounted when procedural times tend to exceed the critical time threshold for severe limb ischemia.

Therefore, MIVT should be offered as a first-line technique to all patients with significant mitral valve disease, even with the need for concomitant treatment of tricuspid valve dysfunction and atrial fibrillation, guaranteeing that a clinical outcome and valve repair quality can be accomplished, at least non-inferior to that of the conventional approach through sternotomy.

Tables

Table 1.

\begin{tabular}{llll}
\hline & Unmatched Patients $(\mathbf{n}=\mathbf{4 3 9})$ & Unmatched Patients $(\mathbf{n}=\mathbf{4 3 9})$ & Unmatched Patie \\
\hline & Sternotomy & MIVT & -value \\
Number & 189 & 250 & \\
Age (years) & $70.0(19.0)$ & $68.0(16.0)$ & 0.850 \\
Gender (male) & $72(38.1)$ & $117(46.8)$ & 0.068 \\
Weight (kg) & $69.0(17.3)$ & $70.0(20.8)$ & 0.319 \\
BSA $\left.\mathbf{( m}^{2}\right)$ & $1.8(0.3)$ & $1.8(0.3)$ & 0.334 \\
Diabetes & $21(12.6)$ & $18(7.2)$ & 0.065 \\
CVA/TIA & $12(6.3)$ & $10(4.0)$ & 0.182 \\
Chronic renal disease & $8(4.2)$ & $2(1.2)$ & 0.191
\end{tabular}




\begin{tabular}{llll}
\hline & Unmatched Patients $(\mathbf{n}=\mathbf{4 3 9})$ & Unmatched Patients $(\mathbf{n}=\mathbf{4 3 9})$ & Unmatched Patie \\
\hline Heart rhythm & & & 0.626 \\
Sinus rhythm & $101(61.6)$ & $164(65.9)$ & \\
Atrial fibrillation & $60(36.6)$ & $82(32.9)$ & \\
PM rhythm & $3(1.8)$ & $3(1.2)$ & $<0.001$ \\
Prior MV surgery & $50(26.5)$ & $12(4.8)$ & $<0.001$ \\
NYHA class & & & \\
NYHA 1 & $7(4.6)$ & $26(12.4)$ & \\
NYHA 2 & $59(39.1)$ & $113(53.8)$ & \\
NYHA 3 & $70(46.4)$ & $63(30.0)$ & 0.187 \\
NYHA 4 & $15(9.9)$ & $8(3.8)$ & \\
LV function (EF) & & & \\
$30 \%$ & $4(2.6)$ & $4(1.6)$ & \\
$30-60 \%$ & $33(21.4)$ & $38(15.2)$ & \\
$60 \%$ & $117(76.0)$ & $208(83.2)$ & \\
\end{tabular}

Data : dichotomous data are given by $\mathrm{n}(\%)$; continuous data are given by median(IQR)

Legend: $\quad \mathrm{BSA}=$ body surfance area; $\mathrm{CVA}=$ cerebrovascular accident; $\mathrm{TIA}=$ transient ischemic attack; $\mathrm{PM}=$ pacemaker; $\mathrm{LV}=$ left ventricular; $\mathrm{EF}=$ ejection fraction

Table 2. Operative data

\begin{tabular}{llll}
\hline & Sternotomy $(n=95)$ & MIVT $(n=250)$ & $p$ value \\
\hline Cardiopulmonary bypass time (min) & $96.0(34)$ & $134.0(42)$ & $<0.001$ \\
Aortic Cross-clamp time (min) & $61.0(26)$ & $87.0(34)$ & $<0.001$ \\
MV repair & & & \\
Posterior leaflet & $30(33.0)$ & $120(48.0)$ & 0.031 \\
Anterior leaflet & $5(5.5)$ & $12(4.8)$ & 0.823 \\
Both leaflets & $13(14.3)$ & $21(8.4)$ & 0.188 \\
Neochordae & $11(12.0)$ & $18(7.2)$ & 0.161 \\
Isolated annuloplasty & $12(13.2)$ & $70(28.0)$ & 0.010 \\
MV replacement & $31(34.1)$ & $27(10.8)$ & $<0.001$ \\
Additional tricuspid surgery & $34(36.2)$ & $45(18.0)$ & $<0.001$ \\
AF Ablation & $14(15.1)$ & $50(20.0)$ & 0.296 \\
\hline
\end{tabular}

Data : dichotomous data are given by $\mathrm{n}(\%)$; continuous data are given by median(IQR)

Legend: $\mathrm{AF}=$ atrial fibrillation

Table 3. Organ-specific biomarkers

\begin{tabular}{llll}
\hline & Sternotomy $(n=95)$ & MIVT $(n=250)$ & Wilk's-Lamda p-value \\
\hline Liver & & & \\
AST (U/1) & & & 0.047 \\
Preop & $25.6 \pm 10.2$ & $26.0 \pm 14.3$ & \\
Day 1 & $67.1 \pm 40.0$ & $81.4 \pm 53.4$ & \\
ALT (U/1) & & & 0.035 \\
Preop & $26.5 \pm 16.9$ & $26.9 \pm 21.5$ & \\
Day 1 & $23.5 \pm 20.8$ & $30.2 \pm 28.4$ &
\end{tabular}




\begin{tabular}{|c|c|c|c|}
\hline & Sternotomy $(n=95)$ & $M I V T(n=250)$ & Wilk's-Lamda p-value \\
\hline $\mathbf{L D H}(\mathrm{U} / \mathrm{l})$ & & & $<0.001$ \\
\hline Preop & $350.7 \pm 119.9$ & $253.1 \pm 106.5$ & \\
\hline Day 1 & $514.7 \pm 174.6$ & $357.6 \pm 145.3$ & \\
\hline \multicolumn{4}{|l|}{ Heart } \\
\hline Creatine kinase $(U / 1)$ & & & 0.002 \\
\hline Preop & $73.9 \pm 54.6$ & $89.7 \pm 61.7$ & \\
\hline Day 1 & $608.3 \pm 634.1$ & $933.0 \pm 795.4$ & \\
\hline CKMB (mg/l) & & & 0.733 \\
\hline Preop & $15.9 \pm 17.8$ & $13.3 \pm 4.61$ & \\
\hline Day 1 & $57.1 \pm 55.9$ & $47.0 \pm 16.7$ & \\
\hline Troponin $(\mathrm{ng} / \mathrm{ml})$ & & & 0.438 \\
\hline Day 1 & $1.8 \pm 3.9$ & $1.2 \pm 1.3$ & \\
\hline \multicolumn{4}{|l|}{ Inflammation and Hematology } \\
\hline CRP (mg/l) & & & $<0.001$ \\
\hline Preop & $1.4 \pm 1.9$ & $1.3 \pm 4.0$ & \\
\hline Day 1 & $5.3 \pm 2.8$ & $4.5 \pm 5.4$ & \\
\hline Day 2 & $177.1 \pm 75.3$ & $17.9 \pm 18.4$ & \\
\hline$\Lambda \varepsilon \cup \varkappa 0 \varsigma \psi \tau \varepsilon \varsigma\left(10^{3} \mu \Lambda\right)$ & & & 0.010 \\
\hline Preop & $7.1 \pm 3.2$ & $6.8 \pm 2.2$ & \\
\hline Day 1 & $10.4 \pm 3.3$ & $11.2 \pm 3.5$ & \\
\hline 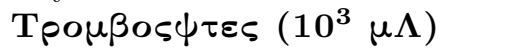 & & & 0.501 \\
\hline Preop & $229.3 \pm 68.1$ & $234.1 \pm 69.2$ & \\
\hline Day 1 & $149.2 \pm 46.7$ & $158.4 \pm 49.6$ & \\
\hline Protrombin activity (\%) & & & 0.006 \\
\hline Preop & $89.9 \pm 11.3$ & $96.9 \pm 13.9$ & \\
\hline Day 1 & $68.1 \pm 9.9$ & $71.2 \pm 10.8$ & \\
\hline APTT (s) & & & 0.045 \\
\hline Preop & $35.9 \pm 5.2$ & $34.8 \pm 5.7$ & \\
\hline Day 1 & $40.0 \pm 8.9$ & $37.2 \pm 5.3$ & \\
\hline Fibrinogen (mg/dl) & & & 0.105 \\
\hline Preop & $401.9 \pm 107.9$ & $379.1 \pm 85.7$ & \\
\hline Day 1 & $293.4 \pm 80.2$ & $287.5 \pm 72.0$ & \\
\hline \multicolumn{4}{|l|}{ Lung function } \\
\hline \multicolumn{4}{|l|}{ Mechanical ventilation } \\
\hline$>24$ hours & $5(5.5 \%)$ & $23(9.5 \%)$ & 0.240 \\
\hline$>48$ hours & $2(2.2 \%)$ & $13(5.4 \%)$ & 0.372 \\
\hline \multicolumn{4}{|l|}{ Blood loss and drainage } \\
\hline Mediastinal loss at 12 hours(ml) & $350 \pm 270$ & $400 \pm 250$ & 0.030 \\
\hline Mediastinal loss at 24 hours(ml) & $510 \pm 305$ & $575 \pm 370$ & 0.038 \\
\hline Blood transfusions(units) & $0(0-9)$ & $0(0-4)$ & 0.492 \\
\hline Blood transfusion volume $(\mathrm{ml})$ & $600 \pm 600$ & $300 \pm 300$ & 0.148 \\
\hline
\end{tabular}

Data: dichotomous data are given by $\mathrm{n}(\%)$; continuous data are given by mean \pm standarddeviation Legend: $\mathrm{AST}=$ aspartate transaminase; $\mathrm{ALT}=$ alanine transaminase; $\mathrm{LDH}=$ lactate dehydrogenase; $\mathrm{CRP}=\mathrm{c}-$ reactive protein; aPTT $=$ activated prothrombin time

Figures

Figure 1. Distribution of MV disease per group 
Figure 2. Survival plot for MIVT and sternotomy

Figure 3. Mitral regurgitation at last echocardiographic follow-up

Figure 4. Freedom from MV-related reoperation per group

Figure 5. Evolution of renal function biomarkers

Creatinine - b) Ureum - c) Glomerular filtration rate

p-value represents the Wilk's-Lamda test

Figure 6. Evolution of CRP

p-value represents the Wilk's-Lamda test

Author contributions

Sophie Missault: concept, data acquisition and analysis, statistics, drafting manuscript

Jérôme Van Causenbroeck: concept, data acquisition and analysis, statistics, drafting manuscript

Korneel Vandewiele: data acquisition and analysis, revision of article

Jens Czapla: data acquisition and analysis, revision of article

Tine Philipsen: data acquisition and analysis, revision of article

Katrien François: data acquisition and analysis, revision of article

Thierry Bové: concept, data acquisition and analysis, statistics, revision of manuscript

References

1. Lamelas J, Nguyen TC. Minimally Invasive Valve Surgery: When Less Is More. Sem Thorac Cardiovasc Surg. 2015;27(1):49-56.

2. Cosgrove DM, 3rd, Sabik JF, Navia JL. Minimally invasive valve operations. Ann Thorac Surg. 1998;65(6):1535-1538; discussion 1538-1539.

3. Gulielmos V, Dangel M, Solowjowa N, et al. Clinical experiences with minimally invasive mitral valve surgery using a simplified Port Access technique. Eur J Cardiothorac Surg. 1998;14(2):141-147.

4. Gammie JS, Zhao Y, Peterson ED, et al. Less-invasive mitral valve operations: trends and outcomes from the Society of Thoracic Surgeons Adult Cardiac Surgery Database. Ann Thorac Surg.2010;90(5):1401-1408, discussion 1408-1410.

5. Dogan S, Aybek T, Risteski PS, et al. Minimally invasive port access versus conventional mitral valve surgery: prospective randomized study. Ann Thorac Surg. 2005;79(2):492-498.

6. Goldstone AB, Atluri P, Szeto WY, et al. Minimally invasive approach provides at least equivalent results for surgical correction of mitral regurgitation: a propensity-matched comparison. J Thorac Cardiovasc Surg. 2013;145(3):748-756

7. Atluri P, Stetson RL, Hung G, et al. Minimally invasive mitral valve surgery is associated with equivalent cost and shorter hospital stay when compared with traditional sternotomy. J Thorac Cardiovasc Surg. 2016;151(2):385-388.

8. Downs EA, Johnston LE, LaPar DJ, et al. Minimally Invasive Mitral Valve Surgery Provides Excellent Outcomes Without Increased Cost: A Multi-Institutional Analysis. Ann Thorac Surg. 2016;102(1):14-21.

9. Iribarne A, Russo MJ, Easterwood R, et al. Minimally invasive versus sternotomy approach for mitral valve surgery: a propensity analysis.Ann Thorac Surg. 2010;90(5):1471-1477; discussion 1477-1478. 
10. Svensson LG, Atik FA, Cosgrove DM, et al. Minimally invasive versus conventional mitral valve surgery: a propensity-matched comparison.J Thorac Cardiovasc Surg. 2010;139(4):926-932

11. Tang P, Onaitis M, Gaca JG, et al. Right Minithoracotomy Versus Median Sternotomy for Mitral Valve Surgery: A Propensity Matched Study.Ann Thorac Surg. 2015;100(2):575-581.

12. Holzhey DM, Seeburger J, Misfeld M, et al. Learning minimally invasive mitral valve surgery: a cumulative sum sequential probability analysis of 3895 operations from a single high-volume center.Circulation. 2013;128(5):483-491.

13. Murzi M, Cerillo AG, Miceli A, et al. Antegrade and retrograde arterial perfusion strategy in minimally invasive mitral-valve surgery: a propensity score analysis on 1280 patients. Eur J Cardiothorac Surg. 2013, 43(6):e167-72.

14. Malvindi PG, Margari V, Mastro F, et al. External aortic cross-clamping and endoaortic balloon occlusion in minimally invasive mitral valve surgery. Ann Cardiothorac Surg. 2018, 7(6):748-754.

15. Suri RM, Schaff HV, Meyer SR, et al. Thoracoscopic versus open mitral valve repair: a propensity score analysis of early outcomes. Ann Thorac Surg. 2009, 88(4):1185-90.

16. Salis S, Mazzanti VV, Merli G, et al Cardiopulmonary bypass duration is an independent predictor of morbidity and mortality after cardiac surgery. J Cardiothorac Vasc Anesth. 2008, 22(6):814-22.

17. Kumar AB, Suneja M, Bayman EO, et al. Association between postoperative acute kidney injury and duration of cardiopulmonary bypass: a meta-analysis. J Cardiothorac Vasc Anesth. 2012, 26(1):64-9.

18. McCreath BJ, Swaminathan M, Booth JV, et al. Mitral valve surgery and acute renal injury: port access versus median sternotomy. Ann Thorac Surg. 2003, 75(3):812-9.

19. Watt DG, Horgan PG, McMillan DC. Routine clinical markers of the magnitude of the systemic inflammatory response after elective operation: a systematic review. Surgery. 2015;157(2):362-380.

20. Paparella D, Rotunno C, Guida P, et al. Minimally invasive heart valve surgery: influence on coagulation and inflammatory response.Interact Cardiovasc Thorac Surg. 2017;25(2):225-232.

21. Vandewiele K, De Somer F, Vandenheuvel M, et al. The impact of cardiopulmonary bypass management on outcome: a propensity matched comparison between minimally invasive and conventional valve surgery. Interact Cardiovasc Thorac Surg. 2020, 31(1):48-55.

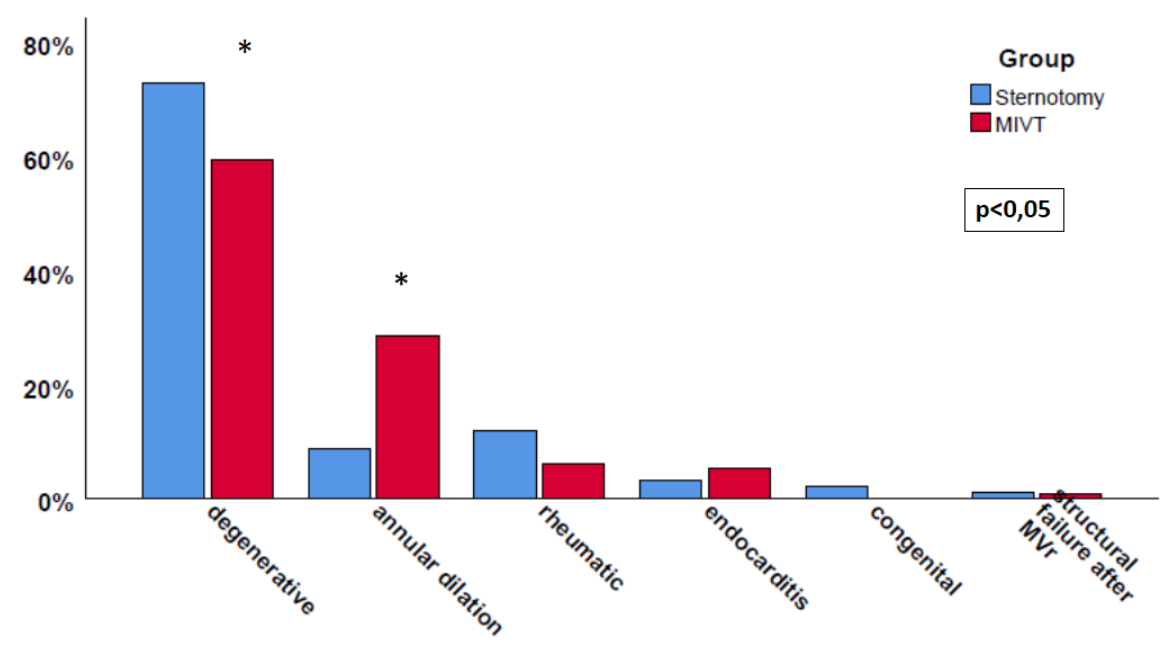



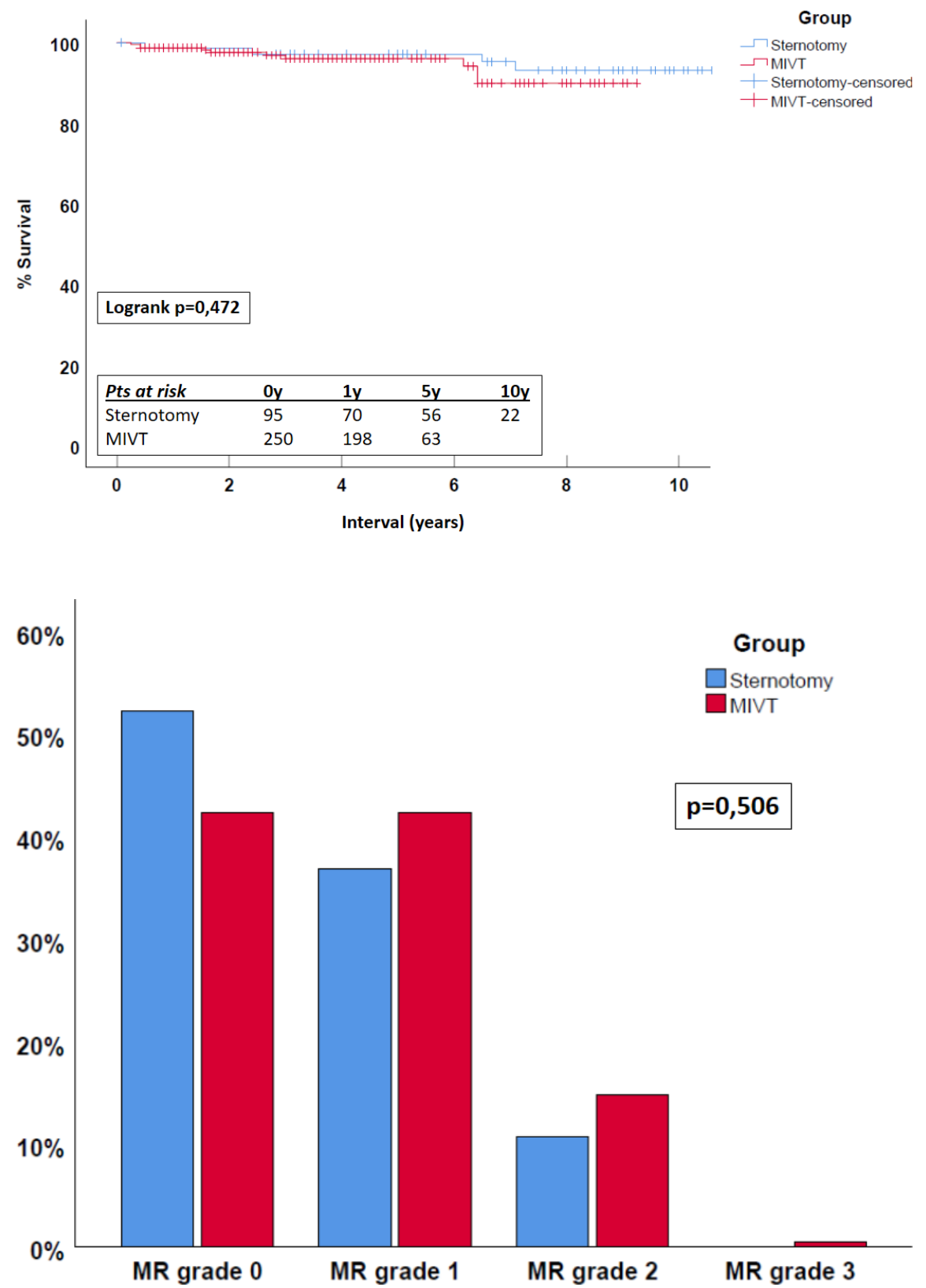

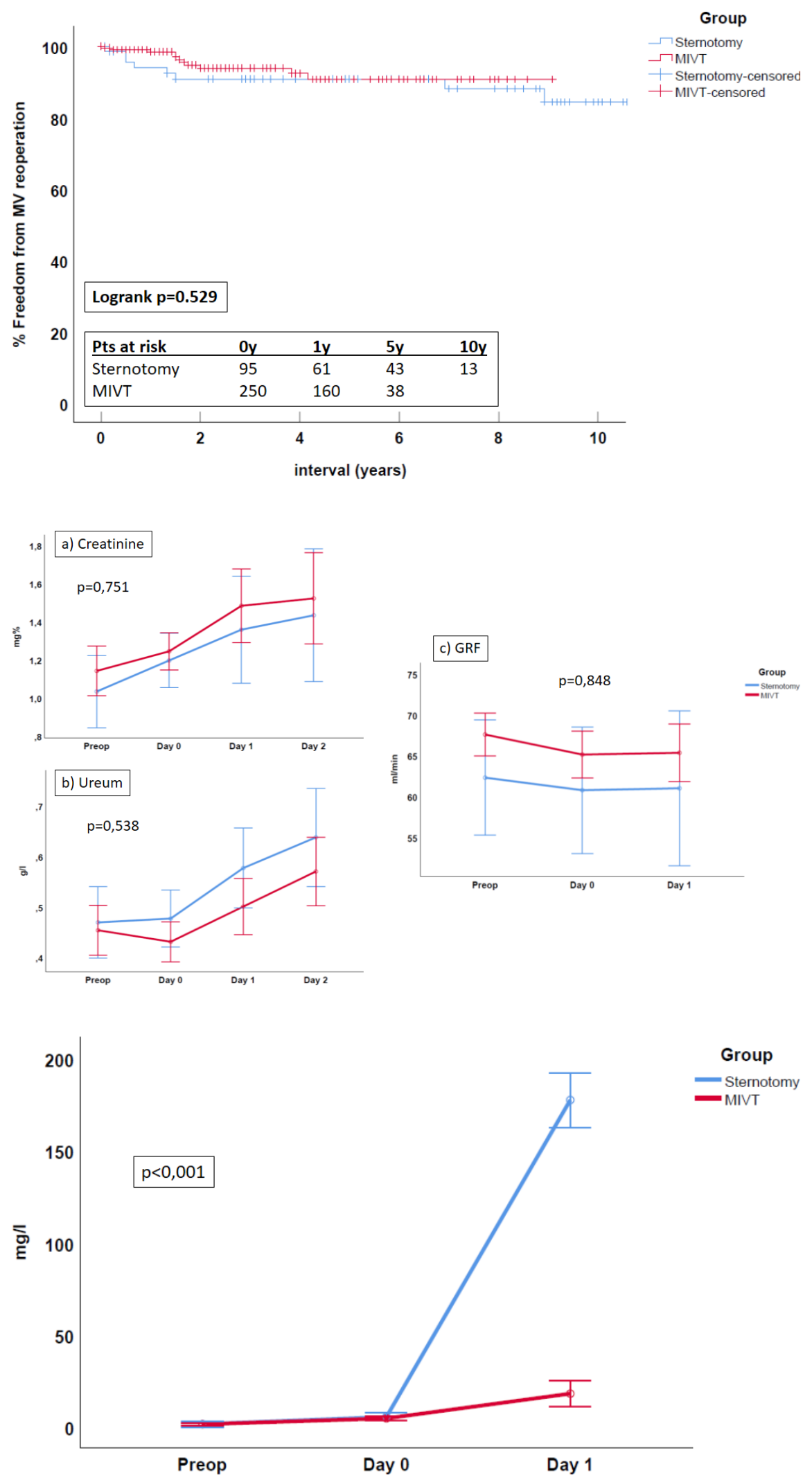